\title{
Radiation doses of digital cephalometric radiography
}

\author{
Radiation doses of indirect and direct digital cephalometric radiography
}

\section{F. Gijbels, G. Sanderink, J. Wyatt, J. Van Dam, B. Nowak and R. Jacobs Br Dent J 2004; 197: 149-152}

\section{Aim}

The aim of this study was to measure organ doses and calculate the effective dose for indirect and direct digital cephalometric exposures.

\section{Material and methods}

Indirect digital cephalometric exposures were made of a Rando ${ }^{\circledR}$ phantom head using a Cranex Tome ${ }^{\circledR}$ multipurpose unit with storage phosphor plates from Agfa and the direct digital (Charge Coupled Device, CCD) exposures were made with a Proline Ceph $\mathrm{CM}^{\circledR}$ unit. Exposure settings were $70 \mathrm{kV}$ and $4 \mathrm{mAs}$ for indirect digital exposures. Direct digital exposures were made with $70 \mathrm{kV}, 10 \mathrm{~mA}$ and a total scanning time of $23 \mathrm{~s}$. TLD700 ${ }^{\circledR}$ dosemeters were used to measure organ doses, and the effective doses were calculated with (effective dose $_{s a l}$ ) and without inclusion of the salivary glands. A pilot study was carried out to compare diagnostic image quality of both imaging modalities.

\section{Results}

Effective doses were $1.7 \mu \mathrm{Sv}$ for direct digital and $1.6 \mu \mathrm{Sv}$ for indirect digital cephalometric imaging. When salivary glands were included in the calculation, effective doses ${ }_{s a l}$ were $3.4 \mu \mathrm{Sv}$ and 2.2 $\mu \mathrm{Sv}$ respectively. Organ doses were higher for direct digital imaging, except for the thyroid gland, where the organ doses were comparable. Diagnostic image quality of indirect and direct digital cephalometric images seemed comparable.

\section{Conclusion}

Effective dose and effective dose ${ }_{s a l}$ were higher for direct digital cephalometric exposure compared with indirect digital exposure. Organ doses were higher for direct digital cephalography. From preliminary data, it may be presumed that diagnostic image quality of indirect and direct digital cephalometric images are comparable.

\section{IN BRIEF}

- This article aims to guide readers in taking the correct purchase/use decision of digital radiographic equipment, based on scientific evidence, by achieving a proper balance between diagnostic quality and radiation dose.

- There is a lower radiation dose to the salivary gland when indirect digital rather than direct cephalometric radiography is carried out.

- Their image quality is comparable for both direct and indirect digital cephalometric radiography.

\section{COMMENT}

This paper, from some of the leading international experts in dental radiology, appears at a time when digital radiography is becoming more widespread and addresses the perennial question of the dose to patients from the different systems available. The authors investigate and compare the two commonly used digital systems, namely CCD direct systems and storage phosphor plate indirect systems for cephalometric exposures. Both systems are clearly explained.

The study was designed to measure organ doses for the two systems using a Rando phantom head with thermluminescent dosemeters positioned in multiple specific anatomical sites throughout the head including the parotid and submandibular salivary glands. Effective doses were calculated for the two digital systems both excluding and including the salivary glands in the calculations. This was important as a number of recent studies have indicated a possible relationship between ionising radiation and salivary cancer. Both calculations showed the effective dose from the direct digital systems to be higher than for the indirect systems, but particularly so when the salivary glands were included in the calculations.

In addition, the authors undertook a pilot study comparing diagnostic image quality of the cephalometric images obtained from the two systems - a crucial aspect from the clinical usefulness of this type of technology - and concluded that they were comparable.

This paper represents a useful addition to the evidence of comparability of different imaging systems and should prove enlightening to all practitioners using or considering using digital imaging.

E. Whaites, Senior Lecturer/Honorary Consultant in Dental Radiology, GKT Dental Institute, London doi:10.1038/sj.bdj.4811529 